\title{
Hollow Tobacco Mosaic Virus Coat Protein Assisted Self-assembly of One-dimensional Nanoarchitectures
}

Jianting Zhang, Ranjith Kumar Kankala, Jingyao Ma, Yihao Zhou, Shi-Bin Wang, Ai-Zheng Chen

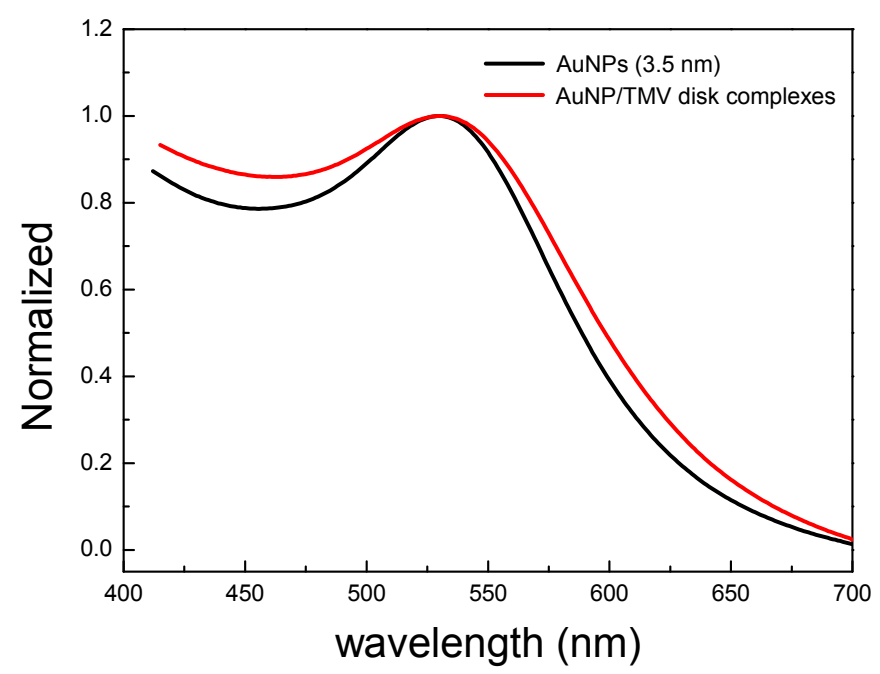

Figure S1. UV spectra of $3.5 \mathrm{~nm}$ AuNP before and after encapsulated in TMV disk.

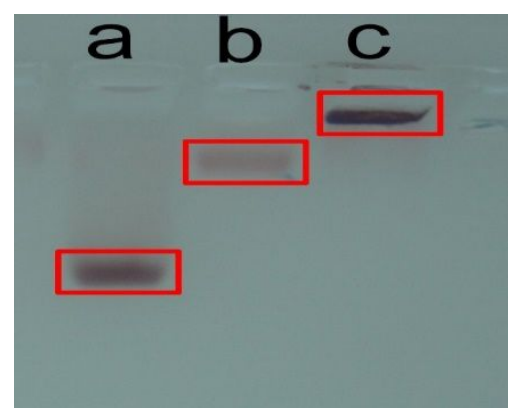

Figure S2. Agarose gel image of free AuNPs (a), TMV-AuNP complex (b) and AuNP nanochains (c). 


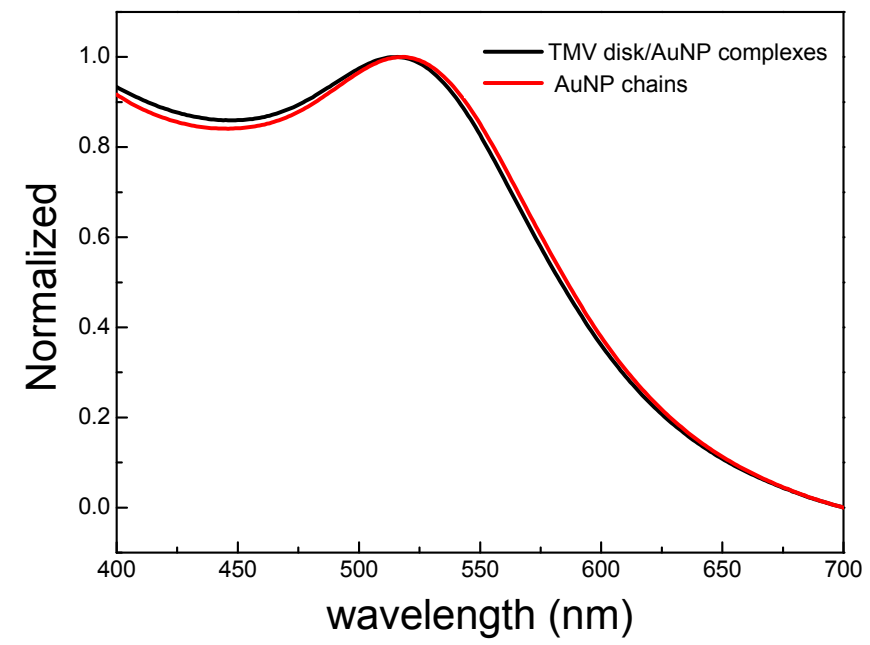

Figure S3. UV spectra of $3.5 \mathrm{~nm}$ AuNPs encapsulated in TMV disks and $3.5 \mathrm{~nm}$ AuNPs inside the TMV fibers.

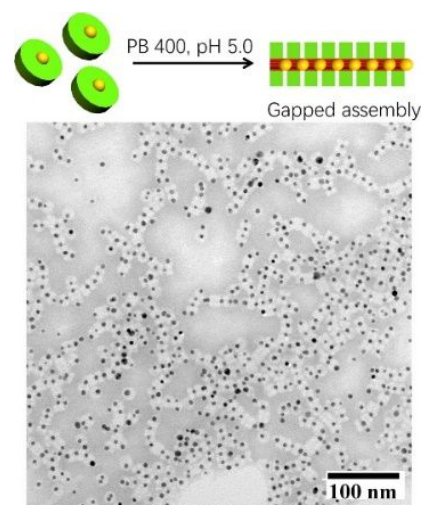

Figure S4. TMV-assisted assembly of $5 \mathrm{~nm}$ AuNP nanochains.

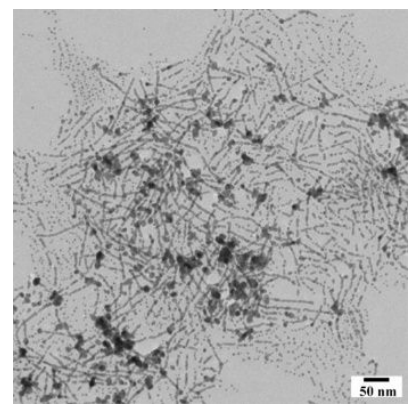

Figure S5. TEM image of TMV-assisted Au nanowires via one-step synthesis. 

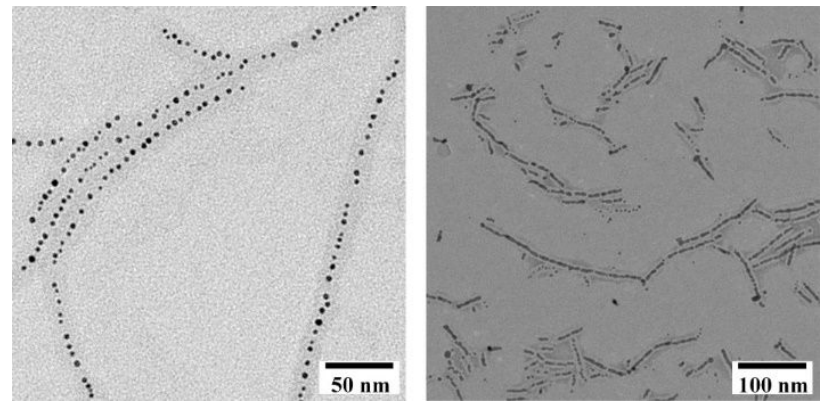

Figure S6. TEM images of TMV-assisted Au nanochains before and after further growth.
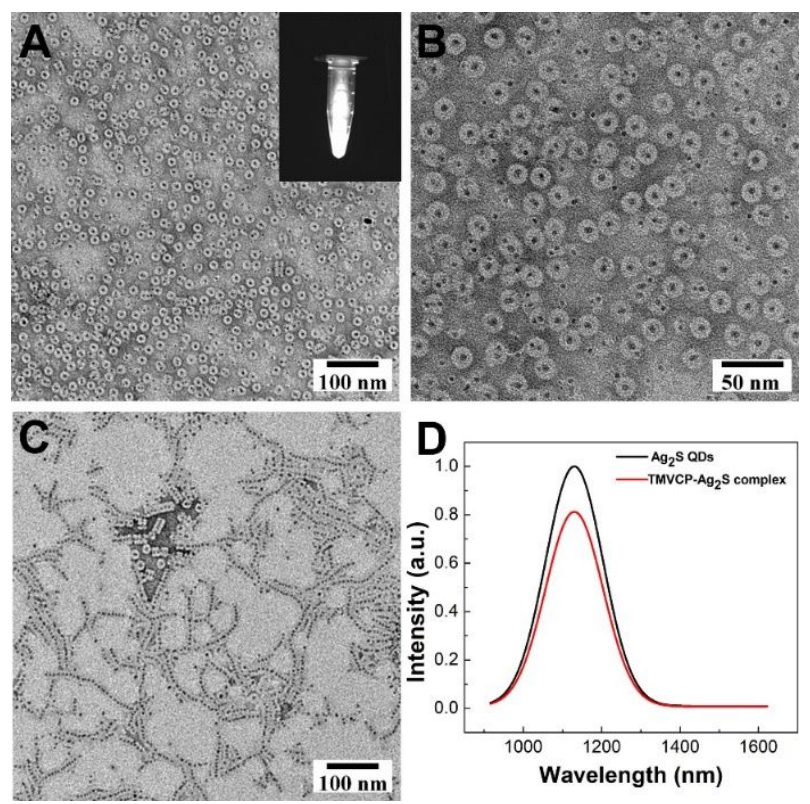

Figure S7. TMV-assisted assembly of $\mathrm{Ag}_{2} \mathrm{~S}$ QDs nanochains. (A, B) TEM images of TMV- $\mathrm{Ag}_{2} \mathrm{~S}$ complexes. (C) TEM image of $\mathrm{Ag}_{2} \mathrm{~S}$ QDs nanochains. (D) Emission spectrum of $\mathrm{Ag}_{2} \mathrm{~S}$ QDs nanochains.
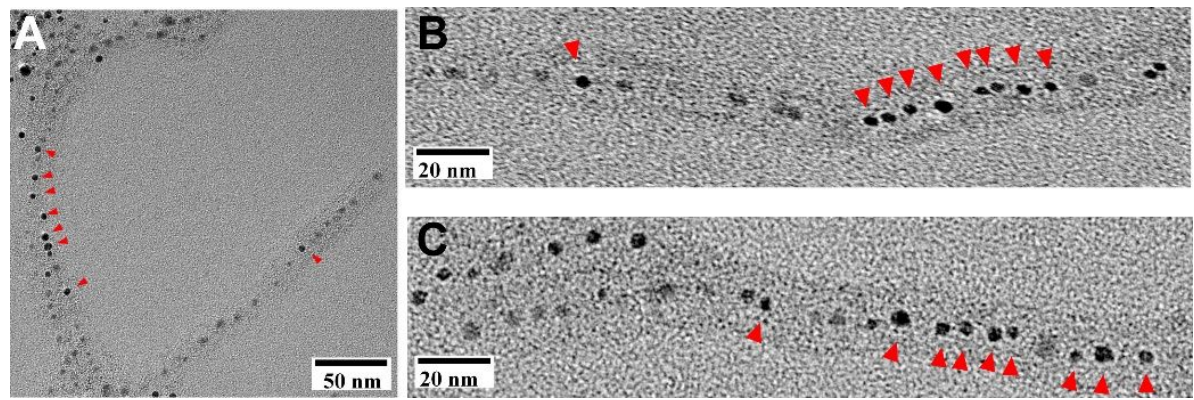

Figure S8. TEM image of nanochains consisting of two different kinds of nanoparticles (AuNPs were signed by red triangles). 

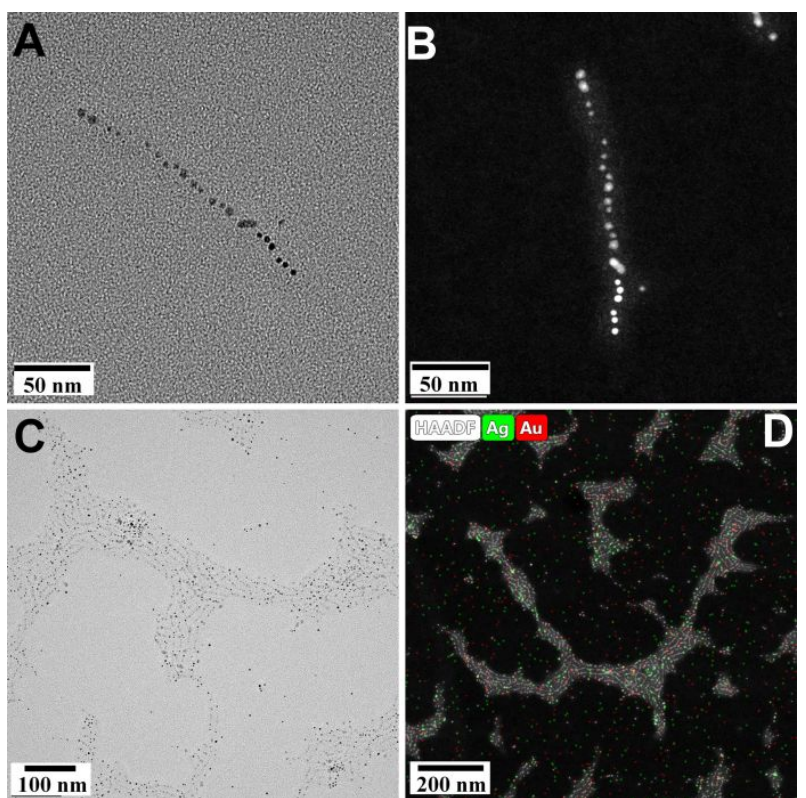

Figure S9. Additional TEM characterization to verify the formation of binary nanochains. (A) Brightfield (BF)-TEM image of binary nanochains. (B) The corresponding HAADF-STEM image of the sample. (C) Low-magnification image of binary nanochains. (D) Element mapping image of the binary nanochains. 